\title{
Molecularly Imprinted Solid Phase Extraction Aiding the Analysis of Disease Biomarkers
}

\author{
Matheus Siqueira Silva ${ }^{a}$, Ana P. M. Tavares ${ }^{b, c}$ (D), Henrique Dipe de Faria ${ }^{a}$, Maria Goreti Ferreira Sales ${ }^{b, c}$, and \\ Eduardo Costa Figueiredo ${ }^{\mathrm{a}}$
}

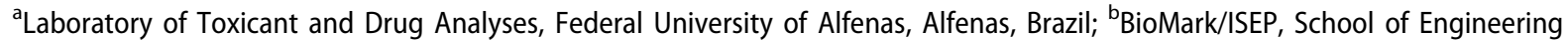
of the Polytechnic School of Porto, Porto, Portugal; 'BioMark/UC, Department of Chemical Engineering, Faculty of Sciences and Technology of the University of Coimbra, Coimbra, Portugal
\end{abstract}

\begin{abstract}
Low concentrations of biomarkers as well as the complexity of biological samples make the clinical diagnoses of several diseases a challenging task. Sample preparation protocols remain a fundamental piece in the puzzle of analytical processes, and smart sorbents including molecularly imprinted polymers (MIPs) have been successfully used in this case. In this review, we depict the state of art for the rational design of MIPs to be used in solid phase extraction of disease biomarkers from biological samples. The topics are divided into (1) strategies for MIP syntheses, (2) setups for sample preparation protocols with MIPs, (3) the applications of these combined principles in the analyses of different classes of disease biomarkers, and (4) remaining challenges and future trends for the application of Molecular Imprinting Technology in sample preparation for clinical diagnosis.
\end{abstract}

\section{KEYWORDS}

Biological samples; disease biomarkers; molecularly imprinted polymers; sample preparation; solid phase extraction

\section{Introduction}

The World Health Organization has defined as biomarkers "any substance, structure, or process that can be measured in the body or in its products, and influence or predict the incidence of outcome or disease." ${ }^{\text {[1] }}$ Thus, the development of procedures to identify/quantify disease biomarkers in complex matrices with minimal interference of concomitant molecules has been target of the study of different researchers around the world. Molecularly imprinted polymers (MIPs) can be highlighted in this case, due to their ability to capture specific molecules in complex matrices such as biological samples (Figure 1(a)). Alvarez-Lorenzo and Concheiro $^{[2]}$ depicts the first stages of development of the MIT field over a historical perspective. Among fields of MIT related to biomarker analyses (Figure 1(b)), MIP-based sensors are the most explored model of application, owing to their portability and ease of use. Some relevant reviews about the theme can be found in literature. ${ }^{[3-5]}$

However, MIPs have been also employed in solid-phase extractions (SPE) to purify complex samples for analyses of disease biomarker. The reviews of Turiel, Tamayo and Martín-Esteban et al., ${ }^{[6,7]}$ Gama and Bottoli ${ }^{[8]}$ and CruzVera et al. ${ }^{[9]}$ reported the use of MIPs to extract small molecules, with a deep discussion about syntheses procedures and SPE protocols. Huang et al., ${ }^{[10]}$ Kataoka and Saito ${ }^{[11]}$ and Pereira et al. ${ }^{[12]}$ reported the MIP applications in bioanalyses of low molecular weight analytes, but in non- comprehensive approaches. In all cases, MIPs are described in a general use, and there are no studies that directly addresses their use in the extraction of disease biomarkers. Additionally, the extraction of high molecular weight biomarkers (proteins and polypeptides) has not been described in depth.

The present review describes the advances in molecularly imprinted solid phase extractions (MISPE) of all types of disease biomarkers (low and high molecular weights), sorting them by biochemical classes (metabolites, hormones, vitamins, peptides and proteins). The SPE strategies related to the analysis of each biomarker class as well as the modern approaches to increase the MIP selectivity are also discussed. MIPs and SPE are discussed in a convergent manner, facilitating the understanding of the advances, remaining gaps and challenges in biomarkers monitoring field.

\section{Main strategies to prepare MIPs for biomarker extractions}

\section{Polymerization techniques}

MIPs used in SPE are usually synthesized by the radical polymerization of a reactional mixture (monomer, template, crosslinker, initiator, and solvent) (Figure 1(a)). Depending on the type of interaction between templates and monomers, molecular imprinting can be covalent, semi-covalent, and non-covalent (most commonly employed). ${ }^{[13]}$ 

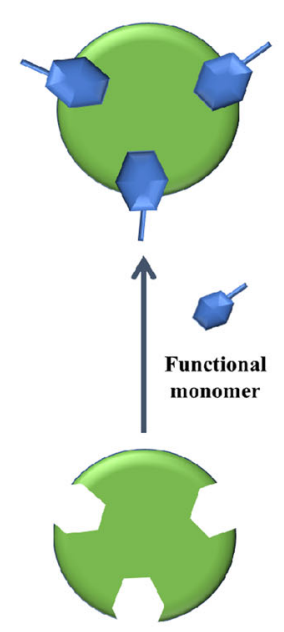

Template molecule

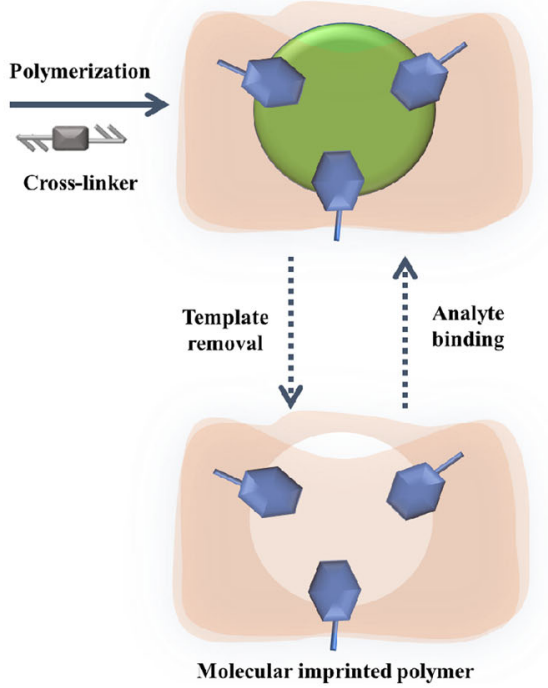

(A)

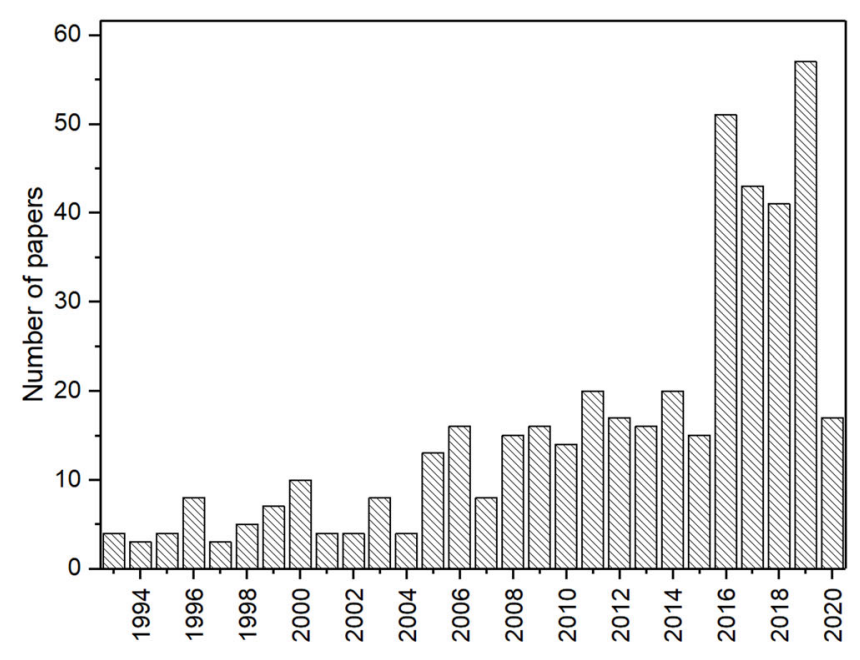

(B)

Figure 1. (a) Schematic representation of molecular imprinting for clinical applications. (b) Number of published papers about molecularly imprinted polymer and disease biomarkers (Web of Science, 04-24-2020, "TS=(((biomolec* OR protei* OR aminoacid* OR clinic* OR hormon* OR marker*) AND diagnos*) AND (imprint* AND polym*))."

Bulk polymerization is the main technique to prepare MIPs for biomarkers to be used in conventional SPE due to the simplicity and greater yield. ${ }^{[6]}$ However, monoliths obtained need to be crushed and sieved, resulting in particles with irregular shapes and sizes. Additionally, some cavities can be destroyed in this procedure, resulting in poor selectivity. ${ }^{[14]}$

Precipitation polymerization is similar to bulk polymerization, but the synthesis is carried out in a more diluted mixture of monomers and crosslinkers. Precipitation polymerization has been selected for the imprinting of biomolecules $^{[15]}$ due to the formation of small spherical polymer beads with a homogeneous distribution of binding sites. ${ }^{[16]}$

In suspension polymerization, the reactional mixture is prepared in polar solvent drops dispersed in water (under agitation), resulting in very homogeneous spheres. ${ }^{[17]}$ If a surfactant is used to stabilize/control the particle size, the procedure is called emulsion polymerization. ${ }^{[16,18]}$ MIP particles prepared by both strategies have presented good performance for recognition in aqueous media. ${ }^{[19]}$

An important point about the selection of the synthesis protocol for biomarker extraction is the particle size. MIP micro/nanoparticles (about from $100 \mathrm{~nm}$ to $10 \mu \mathrm{m}$ ) obtained by precipitation and suspension polymerization are very efficient in dispersive SPE (d-SPE), due to the good interaction with biological samples, as well as the facility to be separated by centrifugation. On the other hand, these particles are not the most suitable for conventional SPE in cartridges or packed columns, mainly due to the high resistance to the flow of these particles. Despite the irregular form, MIPs obtained by bulk have been more useful in these applications.

The structural and chemical complexity of proteins restrain the development of MIPs for these macromolecules by conventional strategies. ${ }^{[20]}$ Surface imprinting has emerged as an alternative approach in the last years, to produce MIP particles (selective to proteins or peptides) around the template molecules immobilized on the surface of a supporting material. ${ }^{[20,21]}$ This leads to the formation of uniformly distributed binding sites near the substrate surface. ${ }^{[16,20]}$ The composition and shape of the support may also compose part of the rational design of the imprinted polymer (see Applications for more information).

For all the procedures described before the control of the growth of polymer chain can result in more homogeneous and selective MIPs. Controlled/living radical polymerization (CLRP) is the main strategy to this control, through chain propagation and termination of the radical species. Reversible addition fragmentation chain transfer (RAFT) and atom transfer radical polymerization (ATRP) are the most important examples of CLRP used to prepare uniform MIPs with small thicknesses in comparison to conventional polymerization. The control of the end-group functionalization is also an advantage, mainly for surface imprinting. ${ }^{[22]}$ The synthesis can be stopped in order to avoid the polymer growth around protein domains that are not defined as the template region. In comparison to traditional imprinting techniques, this procedure allows to precisely project polymers in terms of architecture and molecular weight distribution. CLRP also promote well-controlled copolymers and hybrid materials of organic networks associated with inorganic materials, of special interest in template immobilization/orientation procedures.

\section{Imprinting strategies to improve the MIP selectivity}

Selectivity can be low for some conventional synthesis protocols to obtain MIPs. Thus, strategies have been used to prepare highly selective MIPs for biomarker applications. To bypass some challenges in the recognition of macromolecules, epitope imprinting may be adopted. An epitope is the part of an antigen recognized by an antibody. Peptide 
sequences that match with epitopes can be employed as a surrogate template for larger peptides or entire proteins. Furthermore, the template peptides can suffer modifications that correspond to posttranslational modifications, such as the addition of phosphate, sulfate, or glycosidic groups. This approach makes easier the removal of the template, but diffusion limitations must be addressed in rebinding processes of highly cross-linked polymers. ${ }^{[21]}$ A procedure for epitope selection was proposed by Bossi et al. ${ }^{[23]}$ that consists of an in silico analysis. The protein sequence digestion is simulated, and the generated peptide sequences are screened against the UniProtKB database to authenticate their distinctiveness. MIPs for protein disease biomarkers have often been obtained by this procedure.

Molecularly imprinted cryogels (MICs) have been efficiently used to extract biomarkers from complex samples. ${ }^{[24]}$ These polymers are synthesized under frozen conditions, at a temperature lower than the freezing point of the solvent. ${ }^{[25]}$ MIPs prepared using this strategy present large interconnected pores, providing a short diffusion path, flexibility, and high mechanical strength to bind the biomarker. ${ }^{[26]}$ Additionally, the macropores of cryogels are important to the permeation of high molecular weight disease biomarkers, becoming a very useful strategy in clinical analyses. ${ }^{[24]}$

In MIPs, the interactions between the template and monomers (covalent, semi-covalent, and non-covalent) are crucial to the selectivity. Non-covalent MIPs are the most common because the removal process is very simple, despite the lower selectivity in aqueous media. ${ }^{[27]}$ Metal ions can be introduced as an alternative to enhance template and functional monomer association in water. The monomers and template donate electrons to the unfilled orbitals of the outer coordination sphere of the metal ion, thus forming a ternary complex of fixed spatial orientation. A large variety of natural and synthetic ligands exhibiting donor atoms showed good suitability for the preparation of polymers with good recognition properties. ${ }^{[28]}$

In covalent or semi-covalent MIPs, bonds created between the template and monomers require functional groups such as alcohols (diols), aldehydes, ketones, amines, and carboxylic acids groups, leading to boronic esters, disulfides, ketals, or acetals bonds, among others. In general, these covalent bonds created in the pre-polymerization mixture are reversible, and the template is removed by cleavage. Although covalent bonds offer a high selectivity to MIPs, template removal occurs only under specific conditions (e.g., hydrolysis). [29]

Boronate-based systems were the first approach in covalent MIPs and use boronic acids to reversibly bind cis-diols compounds (including glycoproteins, saccharides, nucleosides, nucleotides) generating stable cyclic esters in an alkaline aqueous solution. ${ }^{[30]}$ These interactions are dissociated in the presence of acidic media. ${ }^{[31,32]}$ Overall, in MIPs synthesized by this strategy, the biomarker undergoes to an orientated interaction. By rational design, the template may be oriented in a way to reproduce the native conformation of the site in the whole molecule, enhancing the speed, accuracy and maximum capacity of the rebinding process. ${ }^{[31,33,34]}$
Restricted access MIPs (RAMIPs) hold a huge unexplored potential for diagnosis purposes. ${ }^{[35]}$ RAMIPs are MIPs able to exclude macromolecules and selectively retain low molecular weight analytes. RAMIPs are externally coated by a hydrophilic surface or proteins ${ }^{[35,36]}$ capable of excluding macromolecules, whereas the analytes are internally captured by different mechanisms. ${ }^{[35,37]}$ These characteristics allow the use of RAMIPs in the extraction of low molecular weight disease biomarkers from protein-rich liquid biological samples, such as human plasma and serum, without prior sample preparation or as few procedures as possible.

\section{Molecularly imprinted solid-phase extraction approaches for biomarker analyses}

\section{Off-line solid-phase extraction}

Although the SPE cartridges are widely used in off-line sample preparation, ${ }^{[6]}$ studies employing MIPs as a sorbent to extract biomarkers are limited, as can be seen in Table 1. However, we believe that the miniaturized SPE cartridges can be a future tendency for biomarker analyses of small sample volumes due to the simplicity, mainly if filled with large MIP particles, that result in low flow resistance to the biological samples.

On the other hand, dispersive SPE (d-SPE) using MIPs has been widely used, due to its simplicity, need of small amount of MIP and solvents, and efficiency in extracting analytes and excluding interfering species. Magnetic MIPs (M-MIPs) have been the main option for d-SPE of biomarkers, given that the particles can be separated by using a simple magnet instead of filtration and centrifugation, ${ }^{[6]}$ in a simple and fast procedure.

At the same line, miniaturized techniques emerged as an alternative to the classical SPE. Extractions are carried out on a reduced scale, requiring less sorbent, sample, and solvents. MIPs have been successfully used in these techniques (fibbers, capillaries, sorptive stirring bars, and well filter plates) to extract biomarkers (Table 1). In Stir Bar Sorptive Extraction (SBSE), a glass capillary is filled with a magnetic material and coated with a MIP. ${ }^{[6,63]}$ SBSE also provides fast and efficient extractions, with low solvent spent and high pre-concentration capacity.

The use of capillaries filled with MIP monoliths is another efficient sample preparation procedure. Synthesis reactions occur inside the capillary having silica as support. ${ }^{[33]}$ Porous MIPs allow sample permeation, thus efficiently extracting the analytes ${ }^{[37]}$ in both on-line ${ }^{[64,65]}$ and off-line procedures. ${ }^{[33]}$ The absence of frits to fix the particle zone is advantageous over the column packed materials by providing lower flow resistance. ${ }^{[65]}$ The non-forced permeation of viscous samples (for example, blood, plasma and serum) through the porous MIP capillary is more difficult, being necessary, in some cases, the use of pumps, as occurs in the on-line procedures commented in the next topic. In addition, the small mass of MIP into the capillary results in low extraction efficiencies, which are compensated by the use of higher sensitive analysis equipment. 


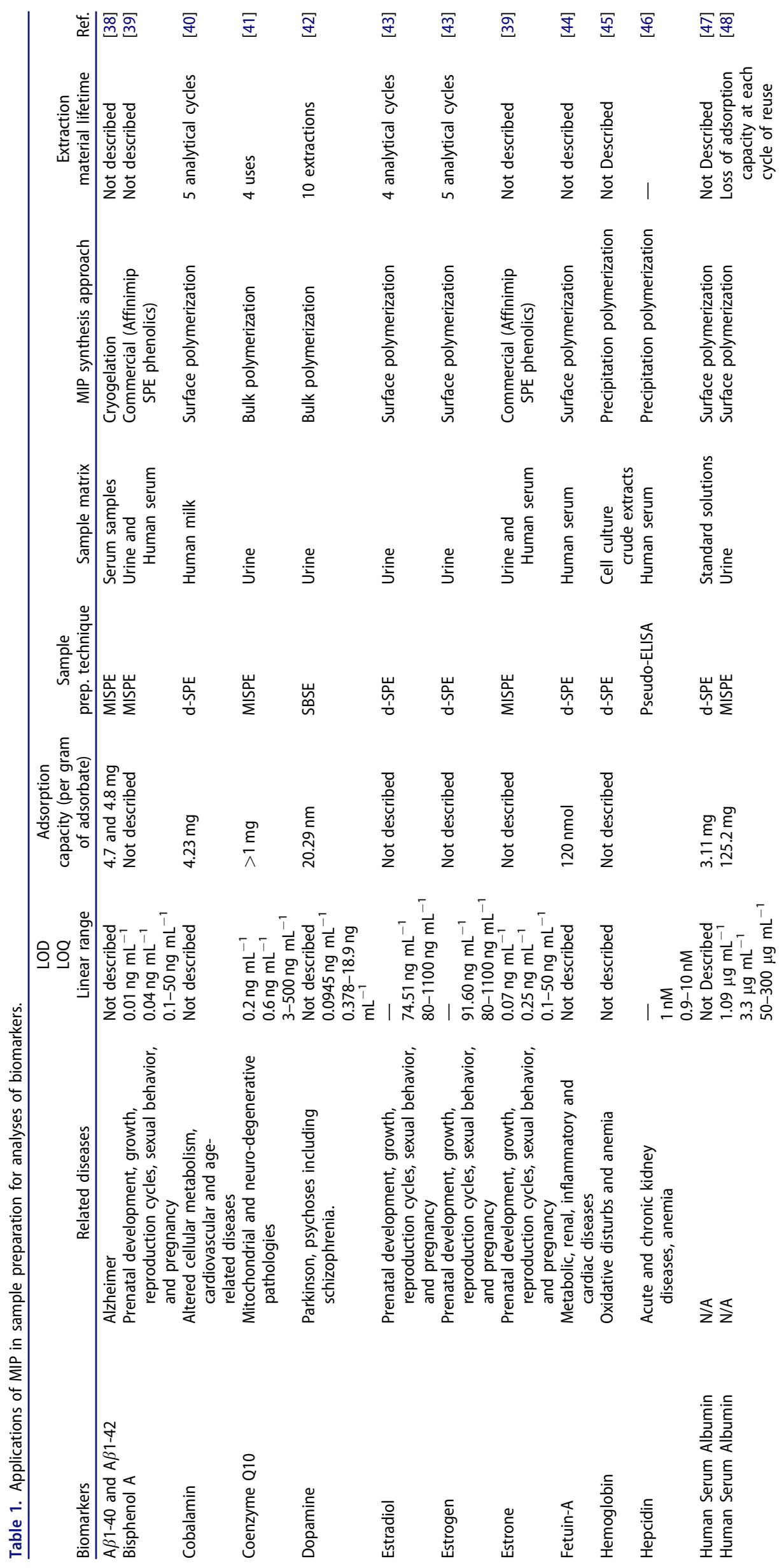




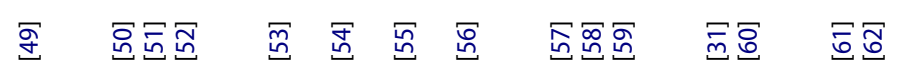

M Mn

IMnIIIIn

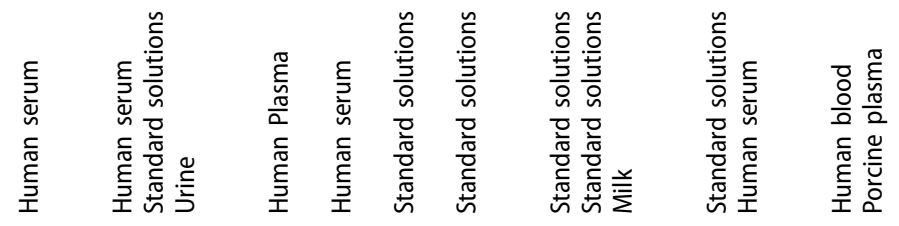

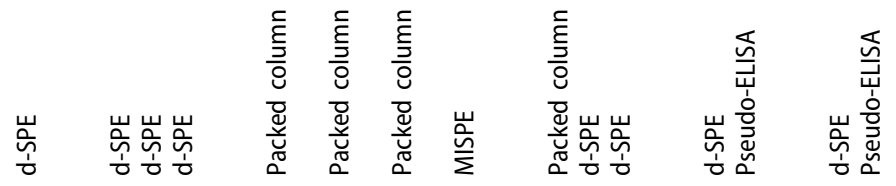

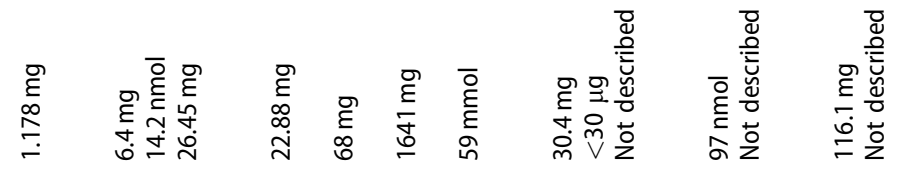

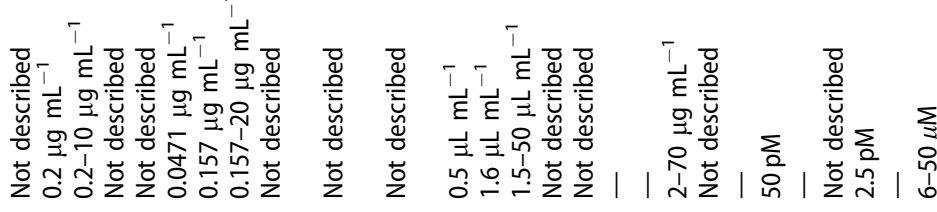

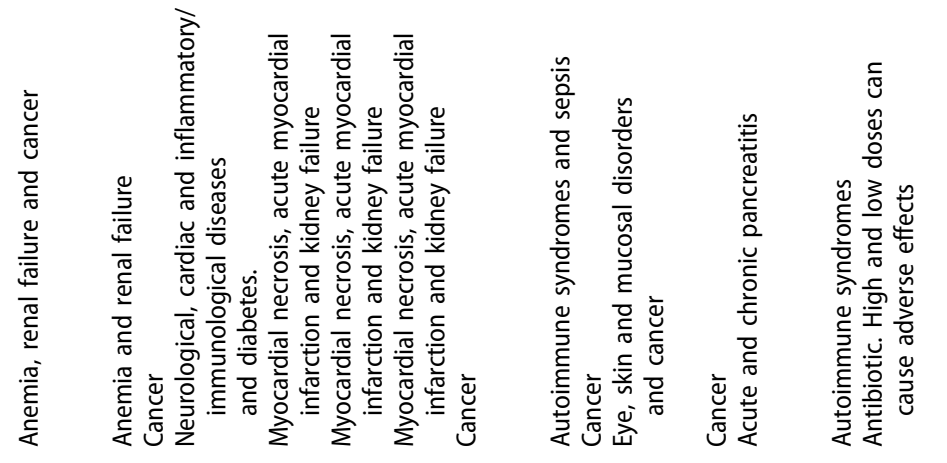

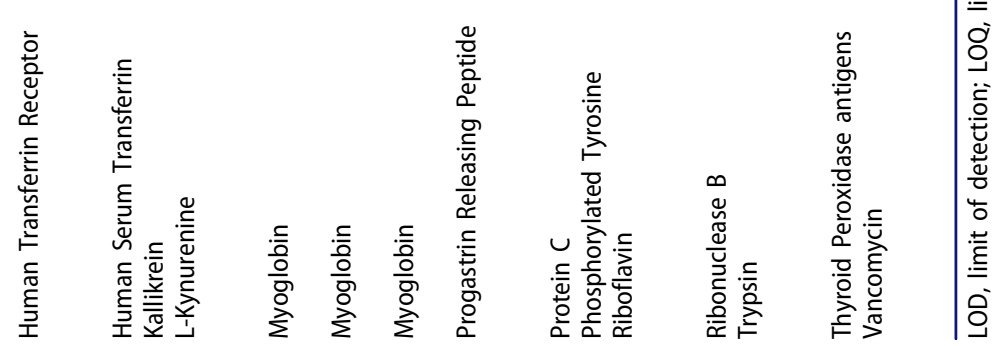


Enzyme-linked immunosorbent assays (ELISA) has been an important approach for disease diagnostics, ${ }^{[66]}$ in which antigens and antibodies are used as selective units. However, MIPs have also been used as recognition units, due to the high cost and low chemical stability of antigens and antibodies, in an approach called pseudo-ELISA. In general lines, pseudo-ELISA is very similar to the conventional SPE. ${ }^{[62]}$ MIPs can be directly polymerized at the bottom of the microplate or grafted on the base of the well after an external polymerization. ${ }^{[67]}$ Different synthesis strategies and target biomarkers can be used, depending on the target analysis. ${ }^{[67,68]}$ On the other hand, the lack of reproducibility and the difficult coating of the microplates wells makes it unfeasible to be used in quantitative analyses. ${ }^{[6,69]}$

In general, a reasonable number of studies employing MIPs in off-line techniques can be found (Table 1), probably because less sophisticated equipment is required. However, sample handling can amplify the experimental errors and decrease analytical frequency.

\section{On-line solid-phase extraction}

On-line extractions have become a worldwide trend due to the improvement of reproducibility, possibility of automation, high analytical frequency and low solvent waste. ${ }^{[2]}$ Monolithic or packed MIP columns/capillaries coupled to chromatographic and electrophoresis systems have been efficiently used for biomarker analyses. In-tube Solid Phase Microextraction (SPME) or column switching Liquid Chromatography (LC) are important examples in which a capillary/column is put in a flow system, operating in extraction and desorption stages. While in in-tube SPME the masses of MIP are less than $5 \mathrm{mg}$, masses of $20-100 \mathrm{mg}$ can be used in column switching. In both cases, the eluates can be analyzed, after leaving the column, by different detection systems, such as ultraviolet UV, ${ }^{[48]}$ electrophoresis, ${ }^{[53]}$ Sodium Dodecyl Sulfate Polyacrylamide Gel Electrophoresis (SDS-PAGE), ${ }^{[70]}$ and Matrix Assisted Laser Desorption Ionization Time Of Flight Mass Spectrometry (MALDITOF-MS), ${ }^{[71]}$ among others. However, the current trend is the use of fully automated systems, including the detector. Although they have successfully applied for other purposes, such as the determination of drugs in human plasma, ${ }^{[72]}$ there are no papers about the determination of disease biomarkers, making this application extremely promising.

Another important extraction procedure is the well filter plates coated by MIPs, in which small sample volumes are used (less than $100 \mu \mathrm{L}$ ). Small MIP masses are inserted into the wells by a vacuum system, and the extraction is carried out for several samples, in some cases using a robotized system that minimizes errors from manual handling. The device can also be easily coupled to Mass Spectrometry (MS) for the analysis. ${ }^{[73]}$ As observed in traditional SPE in cartridges with different sorbents, the particles size is a very important point to be assessed here. MIP nanoparticles request filters with very small pores, which can be easily clogged. The use of larger MIP particles (as those obtained by bulk polymerization), as well as filters with larger pores has been the best option. However, it is important to point out that large particles often present lower adsorption capacity due to the low surface area.

\section{Applications}

MIPs have been excellent sorbents to extract biomarkers for diagnoses of cancer, diabetes, as well as cardiovascular, autoimmune, and infectious diseases. The most important applications of each class of biomarkers (from low weight metabolites to macromolecules) are discussed below.

\section{Metabolites}

The applicability of MIPs for metabolites is still recent (see Table 1) in disease diagnosis. However, the recent advancement of the metabolomics will certainly increase this application in next years. Urine is already the most important biological fluid in this case and water-compatible MIPs will be needed to extract the polar metabolites present in this sample. In this line, an endless arsenal of chemical functions can be easily incorporated into the polymeric matrix, enhancing the selective hindrance.

Dopamine levels are associated to cognition, motor activity, emotion, memory, neuroendocrine regulation and selective attention. Dopamine in urine was targeted by Lei et al.. ${ }^{[42]}$ To do so, an iron wire was inserted into a glass tube and both sides were sealed. A MIP was synthesized on the silylated glass tube, resulting in a monolith (bulk). Dopamine was extracted and analyzed by a LC-fluorescence system. The selectivity of this polymer was tested, and the recovery for dopamine was the highest $(99.2 \%)$, in comparison with L-epinephrine (6.7\%), L-norepinephrine (9.8\%) and 5-hydroxytryptamine (15.9\%). The obtained dopamine concentrations in human urine were correlated with the clinical diagnosis.

L-kynurenine is an endogenous metabolite of the catabolic pathway of L-tryptophan, which can be used as a biomarker for monitoring cancer progression. This metabolite was employed as a template to prepare a MIP monolith, which was used to extract L-kynurenine from human urine $^{[52]}$ by a MISPE protocol, followed by LC analysis. The acidic medium was requested for template dissolution in the porogen mixture, promoting the formation of hydrogen bonds between L-kynurenine and $=\mathrm{O}$ and $-\mathrm{OH}$ residues of methacrylic acid. The polymer was selective to the template (extraction recovery of about $84 \%$ ) in comparison with its structural analogues (L-tryptophan, L-phenylalanine, and L-tyrosine).

A common point between both applications is the use of MIPs obtained by bulk polymerization. Of course, the selectivity of MIPs monolith is easily reached for small molecular weight biomarkers, unlike what happens for proteins, when more elaborated and controlled synthesis protocols are requested. 


\section{Vitamins}

Vitamins are divided in water- and fat-soluble groups, present in almost all type of foods and varied types of biological matrices at low concentrations. The diversity in vitamins chemical properties and structural sizes grant a bunch of opportunities for MIT strategies of preconcentration.

Riboflavin (vitamin B2) is a water-soluble vitamin with the potential to be used in photodynamic therapy for cancer. Its deficiency may be related to eye, skin, and mucosal disorders. Cobalamin (vitamin B12) is important in cellular energy production and DNA synthesis, and its decrease may represent a risk factor for altered cellular metabolism, cardiovascular and age-related diseases. Additionally, its deficiency leads to pernicious anemia. Cobalamin deficits in pregnant women can increase the risk 2-4-fold of neural tube closure defects in fetuses. Li's research group developed M-MIPs by the boronate affinity approach aiming to extract vitamins $\mathrm{B} 2^{[59]}$ and $\mathrm{B} 12^{[40]}$ from cow (commercial) and human milk, respectively. Boronic acid performs reversible covalent bindings with cis-diol-containing compounds (e.g., sugars) at high $\mathrm{pH}$ values, dissociating the formed complexes in acidic $\mathrm{pH}$. This technique allows the use of stronger washing steps, since the strong cis-diol- bindings can maintain the analyte adsorbed. Selectivity was appraised against four cis-diol-containing molecules: vitamins B2 and B6, ascorbic acid, and horseradish peroxidase, and four noncis-diols: quinol, deoxyadenosine, cysteine, and uric acid, thus proving the synergistic selectivity of boronate groups and imprinted cavities. The recovery efficiency was of about $97.1 \%$ in real breast-milk-spiked samples.

\section{Proteins}

Proteomics has developed fast, due to the advances in the MS techniques as well as of strategies to understand the functions of these proteins and interactions between themselves and other biomolecules. A proteome network follows genetic disorders and can adapt to external disturbance, promoting changes in the cellular functional state, and defining specific phenotypes. The post-translational modifications provide a huge variability of proteoforms, and the diversity and abundance can be tracked to a disease-specific phenotype. ${ }^{[74]}$

Even for modern mass spectrometers, analyzing a specific protein in a biological sample is a very difficult task. If the target protein is selectively extracted from a biological fluid, its analysis will certainly be more reliable. This selective extraction can be obtained with MIPs.

The first option to prepare MIPs for proteins is using the entire protein as template. Wen et al. ${ }^{[55]}$ proposed an online SPE based on an imprinted capillary column to extract myoglobin, a myocardial necrosis and infarction biomarker. An internal layer of thermo-responsive MIP presented different behaviors at temperatures below and above $32{ }^{\circ} \mathrm{C}$, resulting in the selective capture and release of myoglobin in presence of other proteins (imprinting factor $=2.33$ ). This temperature influence is the more important advantage of this MIP, given that water, at different temperatures, can be used as eluent, avoiding the use of organic solvents, acids or bases.

An advance in the MIP synthesis with entire proteins as template is the use of chelating agents that orientate the polymerization on specific domains of the protein. More homogeneous binding sites are obtained, improving the selectivity, as demonstrated by thermo-responsive MIP nanoparticles developed by $\mathrm{Xu}$ et al. ${ }^{[51]}$ Kallikrein was used as template, being it a potential biomarker for cancer in processes of cell growth regulation, angiogenesis, invasion, and metastasis. The template orientation was carried out by using p-aminobenzamidine or iminodiacetic acid as chelating agents (Figure 2). Glass beads were functionalized with the affinity ligands and the template:chelating agent complex was formed. Later, the glass beads were loaded in a reactor with the polymerization mixture, and $\operatorname{poly}(\mathrm{N}$-isopropylacrylamide) nanoparticles were formed over the beads. The binding affinity and selectivity tests were performed in a quartz crystal microbalance. MIPs prepared with beads@iminodiacetic acid and beads@p-aminobenzamidine exhibited apparent dissociation constants for kallikrein of $2.6 \times 10^{-10}$ and $4.9 \times 10^{-11} \mathrm{~mol} \mathrm{~L}^{-1}$, respectively. Additionally, no binding occurred with the other serine proteases, attesting the MIP selectivity. The proposed synthesis protocol may work universally for histidine-containing proteins or peptides. Also, peptidic templates can suffer an addition of a His-tag becoming suitable to this chelation.

MICs have been important synthesis protocols to prepare MIPs for proteins (see Imprinting strategies to improve the MIP selectivity), which are structurally more stable at low temperatures, resulting in polymers with more complementary binding sites. As example, Ertürk et al. ${ }^{[53]}$ prepared a MIC column for recognition of myoglobin from human plasma. $\mathrm{N}$-methacryloyl-(L)-tryptophan was used as a functional monomer to provide hydrophobic interactions between the aromatic ring of tryptophan and the hydrophobic residues in myoglobin structure. An on-line SPE was established, and the eluted fractions were assayed by SDSPAGE. The MIPs exhibited distribution constants of 101.42, 13.18, and 7.68 for myoglobin, hemoglobin, and lysozyme, respectively, attesting the selectivity to the template. At the same line, a metal chelation MIC column employing $\mathrm{Ce}$ (III) ions was proposed by Dolak et al. ${ }^{[54]}$ Myoglobin and Nmethacryloylamido antipyrine (MAAP)-Ce(III) were used as template and functional monomer, respectively. Human plasma samples were percolated through the MIC column by pumps, and the detection of myoglobin was performed by UV-Visible-Near Infrared. Extraction efficiency decreased at $\mathrm{pH}$ values higher than 4 , possibly due to the electrostatic repulsion between the $\mathrm{Ce}$ (III) ions and myoglobin. In real serum-diluted samples, the MIP cryogel performed a binding of $95.27 \%$, while non-imprinted substrates performed binding of only $23.46 \%$.

In 2017, Demirci et al. ${ }^{[57]}$ developed a MIC employing metal chelation strategy to determine protein $\mathrm{C}$, a vitamin $\mathrm{K}$ dependent glycoprotein correlated with inflammatory and auto-immune diseases and sepsis. Protein C exhibits 15 


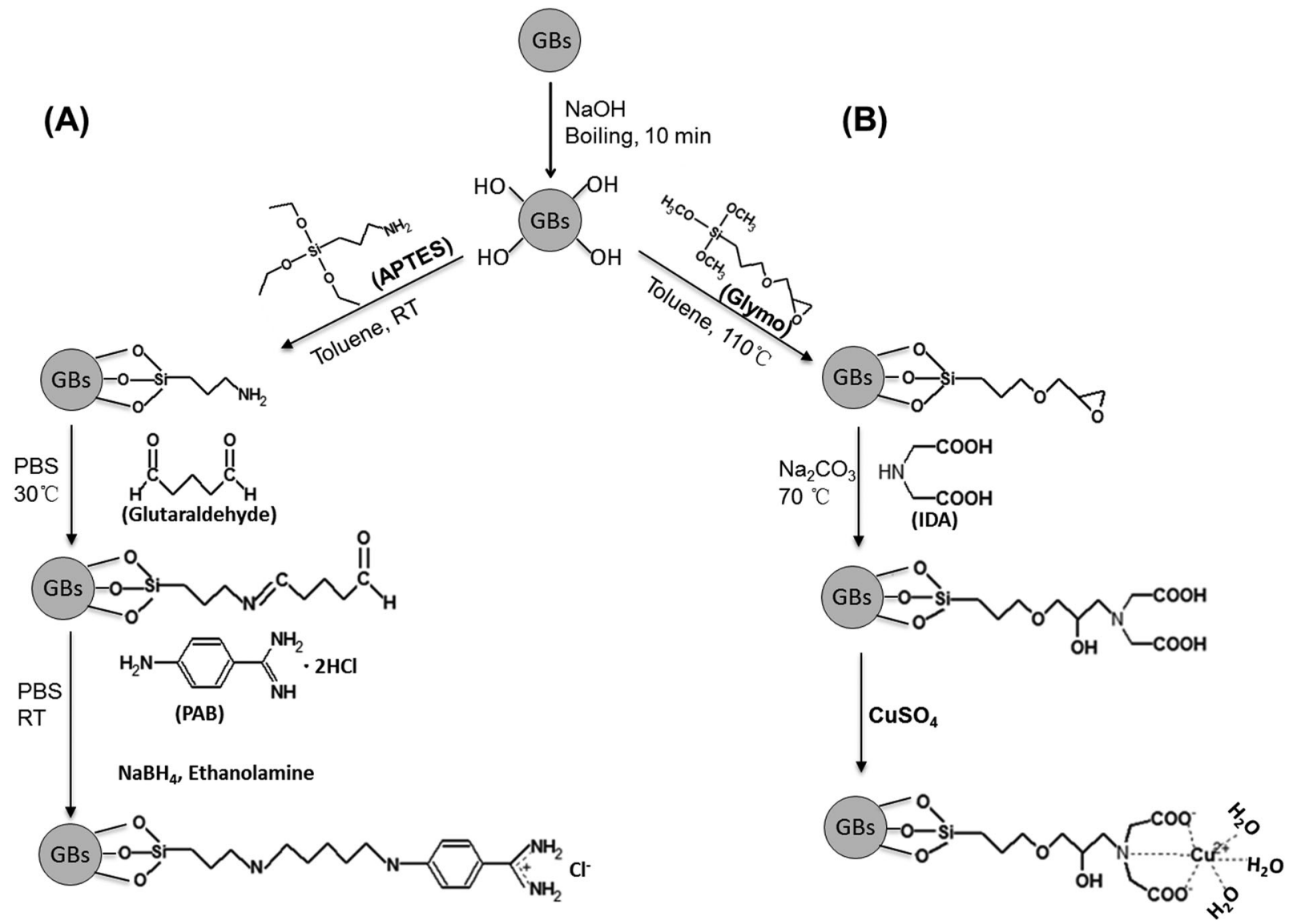

Figure 2. Immobilization of the affinity ligands, (a) p-aminobenzamidine, and (b) iminodiacetic acid on glass beads. Legend: GBs - Glass beads; APTES - (3-aminopropyl) triethoxysilane; PBS - Phosphate buffer solution; RT - Room temperature; IDA - Iminodiacetic acid; PAB - p-aminobenzamidine; Glymo - (3-glycidyloxypropyl)- trimethoxysilane. Reprinted with permission from J. Xu, S. Ambrosini, E. Tamahkar, C. Rossi, K. Haupt, B. Tse Sum Bui, Toward a universal method for preparing molecularly imprinted polymer nanoparticles with antibody-like affinity for proteins, Biomacromolecules. 17 (2016) 345-353. Copyright (2016) American Chemical Society.

surface accessible histidine residues, which can bind to copper ions and coordinate template orientation. The group used standard solutions with known concentrations passing through the monolithic column using a LC system coupled to a UV detector. Chromatographic separation was performed in a linear gradient between the acetate buffer and $\mathrm{NaCl}$. The material demonstrated selectivity when tested against hemoglobin and human serum albumin. The extraction of protein $\mathrm{C}$ was 8.89 and 2.37 times more effective than for human serum albumin and hemoglobin, respectively. Copper chelation of histidine residues yield compatibility with a wide range of protein templates, providing a huge versatility to the proposed method.

Even with the strategies mentioned above, the MIP preparation using entire proteins as templates can result in some MIPs of low selectivity. The high molecular weight of the protein can generate large binding sites, which can be easily obstructed by small molecules during the extraction. As described in "Imprinting strategies to improve the MIP selectivity," the use of epitopes is an important advance to solve this problem. A specific peptide of the target protein is selected as template. These smaller templates are less susceptible to structural changes (such as bends and rotations) during the imprinting process, and the cavities imprinted provide a more trustworthy reproduction of the imprinted site. An example is the work of Bagán et al., ${ }^{[45]}$ in which an epitope of hemoglobin was used as template. Silica nanoparticles were covalently covered with hemoglobin and submitted to tryptic digestion. The obtained nanoparticles were covered with surface epitopes of hemoglobin. A MIP (bulk) was synthesized on the particles and used in d-SPE of hemoglobin from dialyzed crude extracts. The eluates were analyzed by SDS-PAGE, and the selectivity to hemoglobin was attested against bovine serum albumin (BSA) and myoglobin (Figure 3). The imprinted material was able to anchor the whole protein by the recognition of the surface imprinted sites. This strategy can be applied in the diagnosis of disturbances of hemoglobin.

The human transferrin receptor is a transmembrane glycoprotein that can be correlated with breast cancer when at high levels. Liu et al. ${ }^{[49]}$ developed MIPs (bulk) with epitope imprinting approach, in which a tryptic digested peptide of human transferrin receptor was selected as the "surrogate analyte." An important advantage of this strategy is the possibility to use internal peptides as template, instead immunogenic ones of the protein surface, avoiding the interference of antibodies that can compete with the MIP for the binding site. Serum samples were submitted to tryptic digestion and put in contact with MIPs (d-SPE). The bound peptides were eluted and analyzed by LC-MS. MIPs 

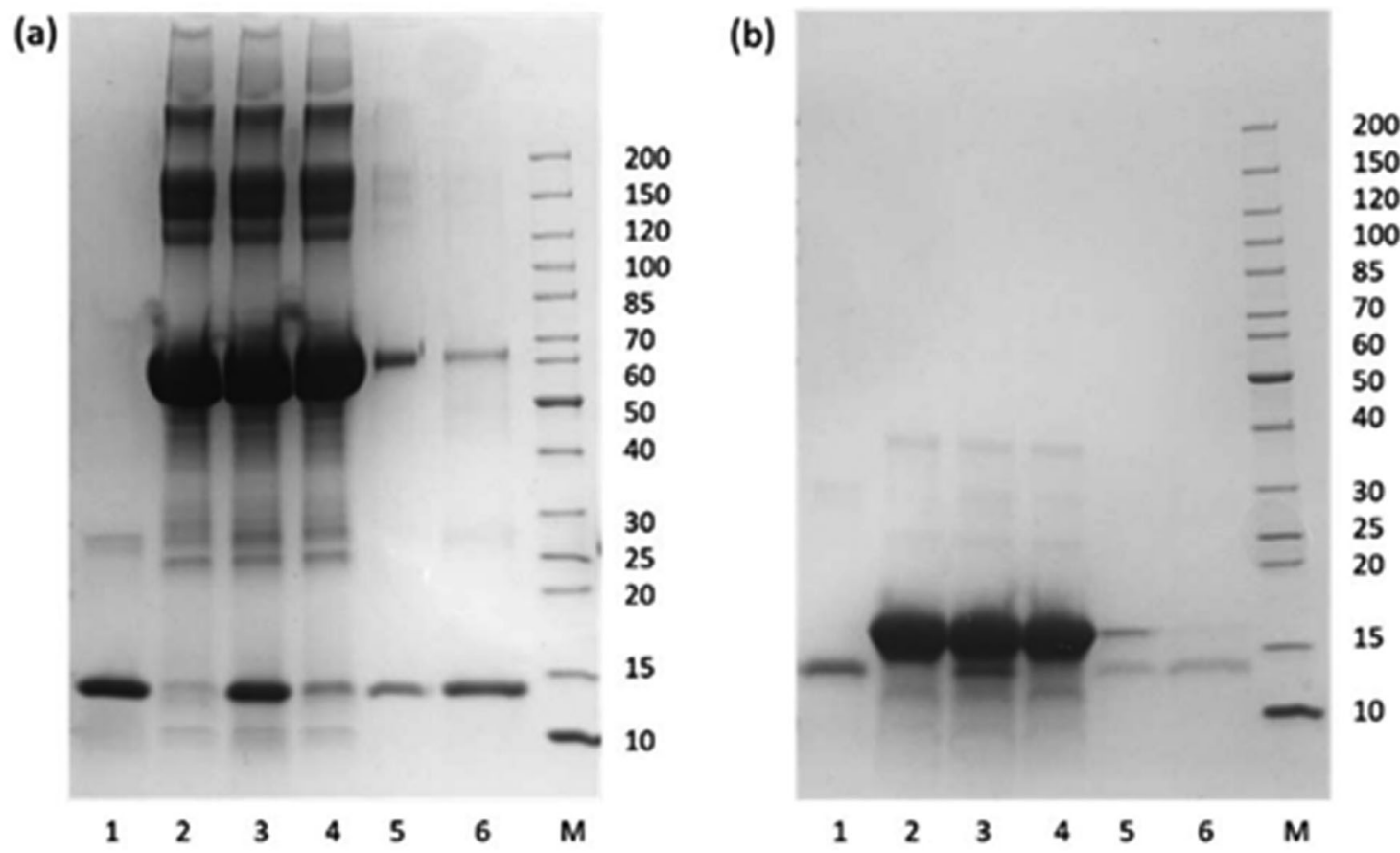

Figure 3. SDS-PAGE of the protein solutions collected from the competitive binding experiments using as interferents BSA (a) and myoglobin (b). Lane 1: Hemoglobin standard; Lane 2: the interfering protein; Lane 3: hemoglobin with the interferent; Lane 4: unbound protein mixture; Lane 5: protein mixture collected in the washing buffer; Lane 6: protein solution eluted from the MIP particles. Reproduced, with minor changes on caption, from Ref. [45] with permission from The Royal Society of Chemistry.

were able to selectively bind to peptides from the transferrin receptor, and a great enhancement on sensitivity was achieved by MS-based proteomics.

Bertolla et al. ${ }^{[50]}$ proposed a solvent-responsive nanoMIPs for the enrichment of human serum transferrin. This protein is involved in the cellular iron uptake process and correlated disturbs. The synthesis was carried out by the precipitation protocol and epitope approach. The template was chosen by epitope fingerprinting. This approach consists in an in silico cleavage of the protein analyte. The generated peptide sequences are screened against the UniProt KB database for rational selection of distinctive and unique peptides. MIPs were able to modify their structure based on the presence of the solvent (acetonitrile), facilitating the desorption step. No digestion was requested before the extractions, and the nanoMIPs bound to human serum transferrin were placed in a target plate for direct analysis by MALDI-TOF-MS. This selective, fast, and simple method had a high pre-concentration capacity of transferrin, raising MALDI-TOF-MS sensibility.

\section{Peptides}

This section was organized with works devoted to extract endogenous peptides biomarkers, naturally present in biological samples, as well as peptides digested from protein biomarkers, and analyzed separately. It is important to point out that in previous section we described the rebinding of whole proteins, even when epitopes were imprinted.

Urraca et al. ${ }^{[38]}$ developed a MIP with a high affinity for $\mathrm{A} \beta 1-40$ and $\mathrm{A} \beta 1-42$, which are $\beta$-amyloid isoforms and Alzheimer's disease biomarkers. The poor solubility observed for the templates required the use of a mixture of DMSO and acetonitrile. The MIP monoliths obtained by the corresponding $\mathrm{N}$-acetylated C-terminal hexapeptide sequences of each isoform as templates were crushed and sieved. The resistance of polymers to chaotropic agents (such as guanidinium chloride) allows affinity enrichment of the amyloid peptides in a fully dissociated form. Peptides were extracted by MIP (conventional SPE) from serum samples and analyzed by urea-SDS-PAGE/immunoblot. High selectivity and recovery were observed for $\mathrm{A} \beta 1-40$ and $\mathrm{A} \beta 1-42$, and the method was able to diagnose Alzheimer's disease.

The template removal step is one of the most critical points in the imprinting process. The selectivity and adsorption capacity of the imprinted polymer for the desired analyte are enhanced by an effective removal process. In other hand, an incomplete removal jeopardizes the selectivity of the polymer and may release the template during elution steps, distorting the results. This phenomena, well known as bleeding effect, is a major issue related to the application of MIT in SPE. Qader et al. ${ }^{[56]}$ described a MIP complementary toward a tryptic signature peptide (epitope fingerprinting) for progastrin releasing peptide, a biomarker for small cell lung cancer. The template sequence was modified in the $\mathrm{N}$ - and $\mathrm{C}$ - terminuses to facilitate the removal of the template, in order to reduce the bleeding effect. The combination of $\mathrm{N}$-(2-aminoethyl) methacrylamide hydrochloride as monomer and divinylbenzene as a crosslinker in a bulk procedure provides a hydrophobic backbone and cavities with ionic groups. Before the MISPE protocol, the protein was digested by trypsin. The eluate from MISPE was dried in nitrogen and resuspended for LC-MS analysis. This model was then adapted in a 384-well filter plate for the screening of molecularly imprinted sorbates. Employing liquid handling robotics, Jagadeesan et al. ${ }^{[73]}$ established a high- 
throughput screening for selection of candidates, synthesis, and washing step optimization.

An epitope approach developed by $\mathrm{Xu}$ et al. ${ }^{[58]}$ was aimed at the enrichment of tyrosine-phosphorylated peptides by d-SPE for MALDI-TOF analysis. Protein phosphorylation is a structural change that is related to the regulation of the biological functions, like the transduction of cell cycles. Phosphorylated tyrosine residues are biomarkers found in tumor proteomes. $\mathrm{Ti}^{4+}$ ions are capable to strongly bind phosphates, so a metal chelation strategy was used. Phenylphosphonic acid was used as a template and $\mathrm{Ti}^{4+}$ immobilized ethylene glycol methacrylate as a functional monomer. Poly-(ethyleneglycol)-diacrylate (a protein repellent reagent) was used as a crosslinker to minimize nonspecific interactions and the polymerization was carried out by precipitation. A tryptic digestion was performed in the sample to obtain fragments of $\beta$-casein. Then the MIPs were inserted into the protein-rich sample, containing the peptides, and incubated for $30 \mathrm{~min}$, centrifuged, washed and the peptides were eluted and analyzed by MALDI-TOF-MS. In this study, the MIPs showed high selectivity and suitable enrichment for phosphorylated tyrosine.

Glycans are complex carbohydrates composed of saccharide units attached to lipids (glycolipids) and proteins (glycoproteins). Their production is regulated by cellular conditions, and glycan alterations can indicate pathological processes. Bie et al. ${ }^{[31]}$ proposed boronate-affinity glycanoriented surface imprinted magnetic nanoparticles to mimic lectin's affinity to glycans and glycoproteins. Ribonuclease B was the targeted glycoprotein. The magnetic nanocores were covered by a layer of boronic acid and glycans were used as templates. Tetraethyl orthosilicate was used to cover the template with a hydrophilic fine-tuned second layer of polymer to avoid unspecific interactions. The thickness of the polymer is a critical element: with a thin layer formed, more "boronic pockets" remains accessible and the greater will be the adsorption capacity of the polymer. The particles were incubated with spiked human serum samples and its tryptic digest, and the bound species could be readily released by eluting with acidic solution. The MIPs were able to bind to other similar proteins: ribonuclease B MIPs adsorbed ribonuclease A (non-glycosylated protein with the same polypeptide chain of ribonuclease B), human serum transferrin (a glycoprotein with two identical silylated glycans) and horseradish peroxidase (containing up to nine identical hybrid glycans). A maximum absorption capacity of $0.097 \pm 0.009 \mu \mathrm{mol} \mathrm{g}^{-1}$ and an imprinting efficiency of $44.8 \%$ were observed. This strategy grants promising materials in glycoproteomics and medical diagnostics.

Bie et al. ${ }^{[44]}$ introduced a dual step in situ enzymatic digestion applying glycopeptides as templates. 3-formyl-phenylboronic acid was used to functionalize the magnetic nanoparticle. The templates (horseradish peroxidase and Fetuin-A) were adsorbed on the particles and two digestions with trypsin and pronase $\mathrm{E}$ were carried out. The use of two proteases combined favor the tuning of the template already oriented on the surface about to be imprinted. This fast, cheap and simple procedure avoid tedious chemical synthesis and purification of templates. MIPs were synthesized on these nanoparticles. The horseradish peroxidase glycan-imprinted NPs exhibited specific adsorption to horseradish peroxidase against ribonuclease $\mathrm{B}$, transferrin, ribonuclease A, and BSA. The Fetuin-A glycan-imprinted NPs were capable to extract Fetuin-A in human serum, excluding abundant interferents as human serum albumin, transferrin, and immunoglobulin G.

Peptide analyses usually focus on signature elements employed as biomarkers. In such a manner, techniques that cover a wide range of compounds with related chemical groups could be applied for screenings over enzymatic digests. The selectivity of these techniques can also be tuned by the strategy of digesting of the biomolecule in ways to expose previously hidden sites, thus amplifying the options of sites to be analyzed in the same target.

MIPs are often prepared in aprotic solvents that minimally interfere in the complex template-monomer during the synthesis. However, practically all applications of MIP are in aqueous medium, and the water interference can minimize the extraction selectivity. Zhao ${ }^{[75]}$ developed a very promising water compatible MIP obtained by micellar imprinting techniques and able to recognize peptides in aqueous solutions. A tripropargylammonium-based surfactant with a methacrylate group in the hydrophobic tail was planned for free-radical polymerization. Tripropargylammonium-based surfactant was dispersed with the template, divinylbenzene (crosslinker), and 2,2-dimethoxy-2-phenylacetophenone (photo initiator) (Figure 4). The first step was the crosslinking of the micelle surface. Large unilamellar vesicles are formed in a $\mathrm{Cu}$-catalysed click reaction between the tripropargylammonium-based surfactant and diazide (a micelle surface cross-linker). Another click reaction was then performed with monoazide to introduce hydrophilic ligands in the micelle. At last, a free radical polymerization was photo-initiated within the surfacefunctionalized micelle by UV light incidence. This nanospace confined polymerization generated high fidelity pockets inside the micelle. This hydrophobic environment provides a background driving force favoring the recognition of hydrophobic side chains in water. Since hydrophobic interactions are the main driving forces in this imprinted nanoparticle, the first examined templates were tryptophan-containing peptides because of their large hydrophobic side chain. Binding studies with glycine-tryptophan peptides showed that even amino acids that lack a hydrophobic side chain could affect the polymer affinity. By employing amphiphilic functional monomers, such as thioronium ions, the imprinted NPs displayed a combination of hydrophobic interactions and hydrogen bonds, boosting selectivity of the formed pockets to acidic side chains of glutamic and aspartic acids.

\section{Hormones}

Unusual variations of free estrogens levels may be related to tumor growth, mainly postmenopausal. Oliveira et al. ${ }^{[43]}$ employed a magnetic-RAMIP covered with bovine serum albumin for the detection of estradiol and ethinylestradiol in urine samples. A polymer of methacrylic acid was formed at 


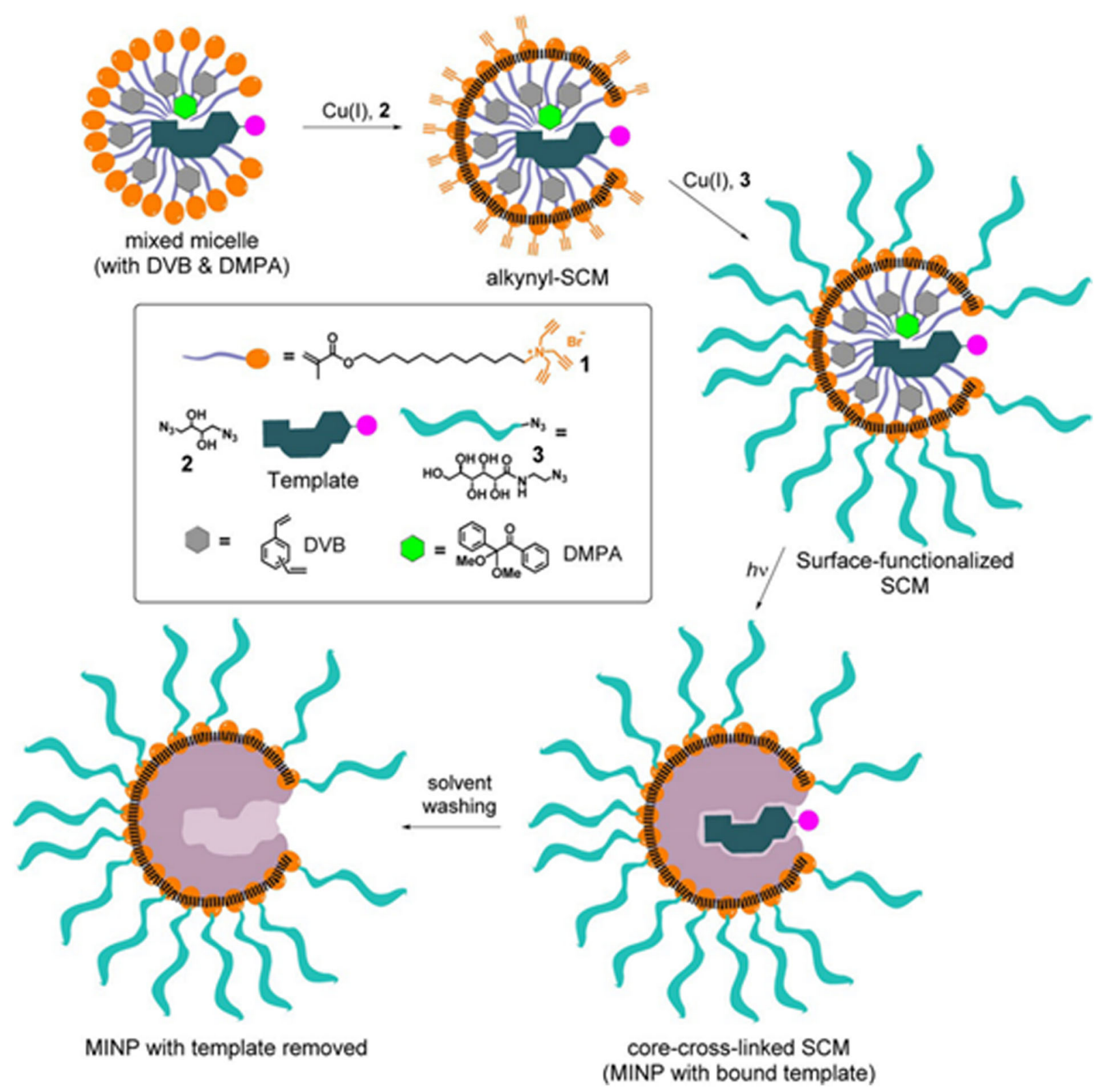

Figure 4. Preparation of molecularly imprinted nanoparticles by surface-core double cross-linking of a template-containing micelle of cross-linkable surfactant. Reproduced from Ref. [76] with permission from The Royal Society of Chemistry.

the surface of magnetic nanoparticles in the presence of estradiol (template). The nanoparticles were coated with the hydrophilic monomers 2-hydroxyethyl methacrylate and glycerol dimethacrylate and then encapsulated with BSA. The BSA layer becomes charged at a $\mathrm{pH}$ different from the BSA isoelectric point, excluding proteins of the sample by electrostatic repulsion. The protein exclusion rate was about $99 \%$, and the recoveries were about $99 \%$ and $96 \%$ for ethinylestradiol and estradiol, respectively.

The RAMIPs were well explored in this paper, simplifying the sample preparation for only one step. We are sure that the use of RAMIPs is a strong tendency in biomarker analyses, because the biomarkers can be extracted from untreated biological samples in very simple procedures like column switching $\mathrm{LC}^{[36]}$ (Figure 5) as well as in SPME. ${ }^{[77]}$ The incorporation of magnetic nanoparticles is another important advantage, being possible carried out a d-SPE instead the conventional SPE in cartridge.
Bousoumah et al. ${ }^{[39]}$ describes an approach for monitoring estrogenic endocrine disruptors. A commercially available MIP SPE cartridge with affinity for phenolic compounds was used to extract 13 analytes: estrone, $17 \alpha$ estradiol, $17 \beta$-estradiol, estriol, $17 \alpha$-ethinylestradiol, diethylstilbestrol, bisphenol A, bisphenol S, 4-n-octylphenol, 4-nnonylphenol, coumestrol, genistein and enterolactone. A LC-MS was validated and further applied to quantify estrogenic endocrine disruptors in human maternal serum, cord serum, and urine samples, with limits of detection ranging from 0.01 to $0.70 \mu \mathrm{g} \cdot \mathrm{L}^{-1}$ (Table 1 ).

\section{Depletion of interfering species}

Most papers about MIPs reported the selective capture of an analyte of interest from a sample. However, the use of MIPs to capture interferents is a new and promising approach. In 


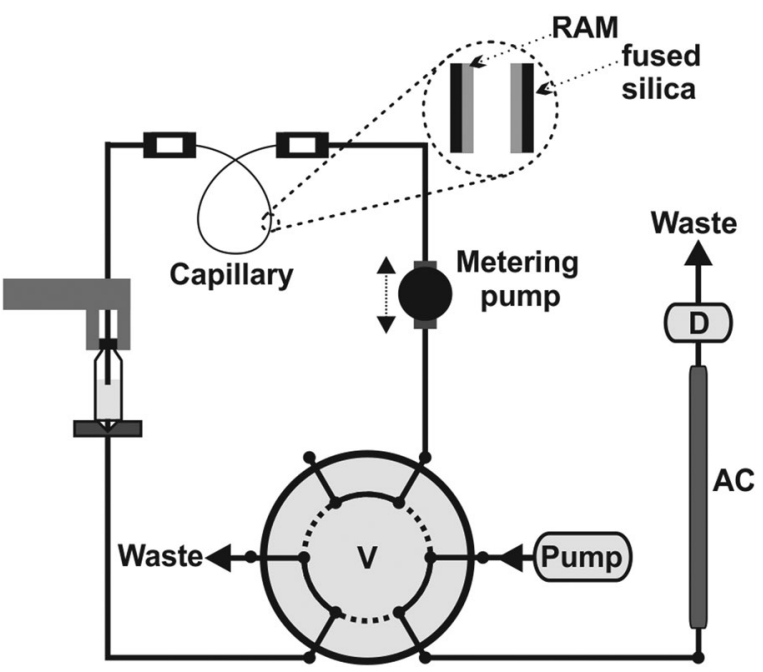

Figure 5. In-tube SPME liquid chromatography system using a RAM capillary in the extraction position (V: valve; AC: analytical column), adapted from Anal. Chim. Acta, 959, H.D. de Faria, L.C. de C. Abrão, M.G. Santos, A.F. Barbosa, E.C. Figueiredo, New advances in restricted access materials for sample preparation: A review, 43-65 (2017), with permission from Elsevier.

this way, Yang et al. ${ }^{[71]}$ developed a MIC by using human serum itself as template. Abundant proteins were preferentially imprinted in the MIC, determining the polymer selectivity. The material was conditioned in LC columns and a human sample flowed through it. The remained sample was analyzed by SDS-PAGE (Figure 6). The polymer was able to deplete abundant serum components as albumin, serotransferrin, and most globulins. The depleted samples exposed lower abundance proteins that were enzymatically digested and identified by MALDI-TOF-MS analyses.

This revolutionary application of MIPs can be extremely useful in the discovery of biomarkers. After all, abundant proteins camouflage biomarkers and important information about the presence or changes in concentration of these biomarkers can be lost.

\section{Pseudo-ELISA}

In pseudo-ELISA protocols, the biological antibodies are exchanged by MIPs, which are used as anchors in the microplate for the analyte immobilization (a MISPE). Additionally, MIPs can also be used as a transducing unit when fluorescent monomers are used in the synthesis.

$\mathrm{Xu}$ et al. ${ }^{[60]}$ developed core-shell imprinted fluorescent nanoparticles used in an immunoassay for the determination of trypsin in human serum. Trypsin is involved in a regulatory system to prevent pancreatic autodigestion and can be followed as a biomarker for acute and chronic pancreatitis. Thermoresponsive MIP nanoparticles were synthesized by a protocol similar to the one described previously (Metabolites ${ }^{[51]}$ ), using trypsin as a template. The nanoparticles produced over the beads were conjugated with fluorescein isothiocyanate before the release. The binding sites were protected by the solid support during the conjugation. A microplate functionalized with $p$-aminobenzamidine was used to capture trypsin. For the equilibrium assay, the polymer was incubated in wells functionalized with trypsin. The

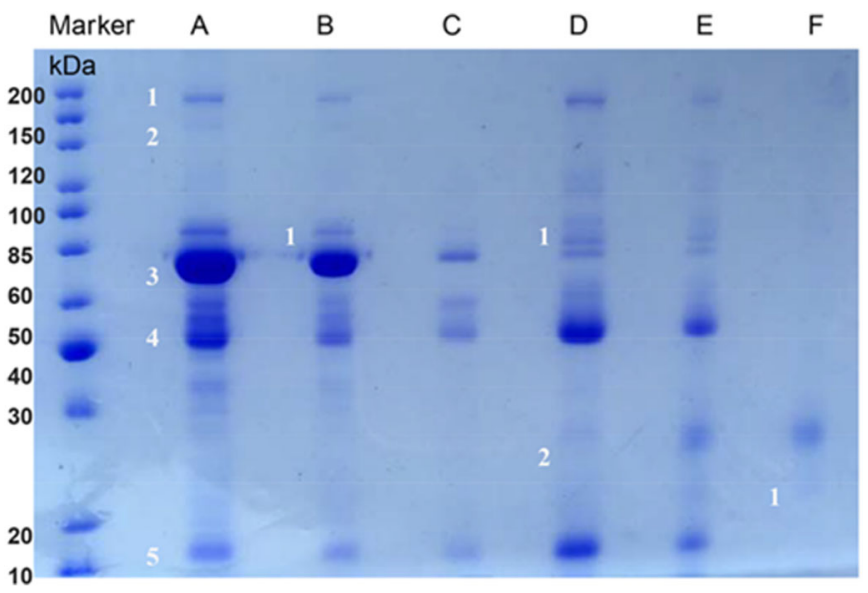

Figure 6. SDS-PAGE of serum eluates from different columns. (a) Original serum, (b) NIP, (c-f) MIPs prepared with different monomers. Proteins identified: A1, $\alpha$-2-macroglobulin; A2, Ig $\gamma$ chain C region; A3, albumin; A4, Ig heavy chain V-III region; A5, Ig $\kappa$ chain V-III region; $\mathrm{B} 1, \lg \alpha-1$ chain $\mathrm{C}$ region; $\mathrm{D} 1, \lg \mu$ chain C region; D2, Serine/threonine- protein kinase; F1, $\alpha$-1-acid glycoprotein. Reprinted with permission from C. Yang, Y.-R. Liu, Y. Zhang, J. Wang, L.-L. Tian, Y.-N. Yan, W.-Q. Cao, Y.-Y. Wang, Depletion of abundant human serum proteins by per se imprinted cryogels based on sample heterogeneity, Proteomics. 17 (2017) 1600284. Copyright (2017) American Chemical Society.

binding isotherms showed a linear trend of binding almost $100 \%$ for the tested concentrations. The selectivity was tested against kallikrein, thrombin, cytochrome C, and human serum albumin, and little cross-reactivities were observed. By reaching very low concentrations, this method allows dilution of the sample to reduce background signals.

Cenci et al. ${ }^{[46]}$ took a closer look at the preparation and stability of the pseudo-ELISA plates. Fluorescent MIPs of acrylamide, methacrylic acid, and acryloxyethyl thiocarbamoyl Rhodamine B were prepared by precipitation polymerization. Different strategies of particle deposition on different supports and the stability after subsequent washings were evaluated by measuring the fluorescence of the MIP nanoparticles. The procedure was tested with a nanoMIP for hepcidin, a protein known as a key regulator of iron homeostasis. Hepcidin levels are important predictors of acute kidney injuries, chronic kidney diseases, and may help with differential anemia diagnosis. The assay was able to measure hepcidin at $1 \mathrm{nmol} \mathrm{L}{ }^{-1}$ in spiked serum samples, with high recovery and good reproducibility. The authors highlighted that the immobilization strategies between the plate and MIP are important to minimize the loss of particles during the washing steps.

Polymers are less susceptible to variations on binding sites promoted by proteases and broad ranges of temperature and $\mathrm{pH}$. Moreover, the possibilities of assimilating fluorescent, magnetic, thermoresponsive properties, and the tunable polarity of polymeric chains furnishes a vast universe of strategies to override the limitations of traditional ELISA platforms.

\section{Limitations, outlook and future perspectives}

The advances in the "omics" field brought new biomarkers into the scope of clinical diagnosis, which in turn created new challenges to the analytical perspective. The 
concentrations of these new biomarkers in real samples are getting lower when compared to the usual analytes. Further purification and pre-concentration protocols are desirable to boost the sensitivity of current methods. In fact, the evolution of these protocols aids on development of "omics" field and a "vicious circle" of MIT development is stablished. This allows the analysis of low concentration analytes with simpler transduction units and enables these new biomarkers to take their place in the analytical market.

Sensors are based on a recognition unit associated with a recognition element or indirect measurements. These portable units allow a fast detection in field, with no need of laboratorial infrastructure. However, they generally lack amplification and may require a very sensitive transducers. This scarce amplification may contribute with the occurence of false results. For a daily laboratorial routine, the robustness is imperative. Despite the practicality of MIT sensors, the tenacity of results obtained on chromatographs, with a broad range of transducers, are still commercially attractive.

Sample preparation steps are pivotal tools for the qualitative and quantitative determination of biomarkers in complex matrices. The MISPEs fits in this field and provides environment-friendly protocols, since it: (1) allows miniaturization and reduces the amount of solvent and sample needed as well as (2) replacing biological recognition elements (e.g., antibodies and enzymes) by artificial ones of simpler manufacture. The combination of sample preparation and MIP bring together the benefits of both as well as links the challenges between them: (1) poor template removals hinder the yield of specific anchoring sites and may result in leakage during the purification steps, (2) synthetic conditions and template constitution generally make it difficult to reproduce the specificity of biological receptors, and (3) nonspecific interactions may contribute to co-extraction of interfering compounds and weaken sensitivity.

MIP-based sample preparation protocols also take advantage of the recent developments in terms of rational template design for biomacromolecules imprinting. The creation of new strategies raises the number of possible targets (e.g., different biochemical classes) and the use of new functional monomers capable of complexing with the template more strongly improve the selectivity. Protocols to obtain magnetic MIPs can also be highlighted, due to relevant advance in their use in d-SPE of biomarkers.

The use of moderns restricted access technologies can also be highlighted as a promising tendency to prepare RAMIPs for the extraction of low molecular weight analytes from untreated biological fluids. The proteins are excluded, avoiding the need of time-consuming steps of deproteination. This property can also benefit the analysis of inorganics as metals. On-line procedures based on column switching LC with RAMIPs will certainly stand out in the coming years, due to the possibility of injecting raw samples in the LC-MS equipment. The suitability for automation and lab-on-chip models can also motivate the development of sensing platforms that perform sample treatment and detection (as the pseudo-ELISAs).
MIT models for sample preparation are commercially available in product lines (e.g., SupelMIP, AFFINIMIP, Affinilute) for environmental, clinical and forensic purposes. The catalogs count, for example, with options for the extraction of phenolic compounds, beta-blockers or even significant drugs of abuse from biological fluids. This field tends to evolve indefinitely since it presents well-defined challenges in terms of the discovery of new biomarkers, and day-by-day, new developments of sample preparation and MITs open new bases of possible combinations to fulfill the needs of the clinical and other markets.

\section{Funding}

The authors are thankful to the Fundação à Pesquisa do Estado de Minas Gerais (FAPEMIG, Belo Horizonte, Brazil); the Conselho Nacional de Desenvolvimento Científico e Tecnológico (CNPq, Brasilia, Brazil) and the Coordination for the Improvement of Higher Education Personnel (CAPES, Brasilia, Brazil) for financial support (process 88881.133746/2016-01) and the scholarship funding for Matheus Siqueira Silva/PDSE/ Processo no. $\{88881.133746 / 2016-01\}$.

\section{ORCID}

Ana P. M. Tavares (ID) http://orcid.org/0000-0003-2868-3037

\section{References}

[1] World Health Organization. Biomarkers in Risk Assessment: Validity and Validation. World Health Organization \& International Programme on Chemical Safety: Geneva, 2001, p 238.

[2] Handbook of Molecularly Imprinted Polymers. Alvarez-Lorenzo, C., Concheiro, A., Eds.; Smithers Rapra: Shawbury, UK, 2013.

[3] Lin, Z. T.; Demarr, V.; Bao, J.; Wu, T. Molecularly Imprinted Polymer-Based Biosensors: For the Early, Rapid Detection of Pathogens, Biomarkers, and Toxins in Clinical, Environmental, or Food Samples. IEEE Nanotechnol. Mag. 2018, 12, 6-13. DOI: 10.1109/MNANO.2017.2779718.

[4] Selvolini, G.; Marrazza, G. MIP-Based Sensors: Promising New Tools for Cancer Biomarker Determination. Sensors 2017, 17, 718. DOI: $10.3390 / \mathrm{s} 17040718$.

[5] Frasco, M.; Truta, L.; Sales, M.; Moreira, F. Imprinting Technology in Electrochemical Biomimetic Sensors. Sensors 2017, 17, 523. DOI: 10.3390/s17030523.

[6] Turiel, E.; Martín-Esteban, A. Molecularly Imprinted Polymers for Sample Preparation: A Review. Anal. Chim. Acta. 2010, 668 , 87-99. DOI: 10.1016/j.aca.2010.04.019.

[7] Tamayo, F. G.; Turiel, E.; Martín-Esteban, A. Molecularly Imprinted Polymers for Solid-Phase Extraction and Solid-Phase Microextraction: Recent Developments and Future Trends. J. Chromatogr. A. 2007, 1152, 32-40. DOI: 10.1016/j.chroma.2006. 08.095.

[8] Gama, M. R.; Bottoli, C. B. G. Molecularly Imprinted Polymers for Bioanalytical Sample Preparation. J. Chromatogr. B. Analyt. Technol. Biomed. Life. Sci. 2017, 1043, 107-121. DOI: 10.1016/j. jchromb.2016.09.045.

[9] Cruz-Vera, M.; Lucena, R.; Cárdenas, S.; Valcárcel, M. Highly Selective and Non-Conventional Sorbents for the Determination of Biomarkers in Urine by Liquid Chromatography. Anal. Bioanal. Chem. 2010, 397, 1029-1038. DOI: 10.1007/s00216010-3476-3.

[10] Huang, S.; Chen, G.; Ye, N.; Kou, X.; Zhu, F.; Shen, J.; Ouyang, G. Solid-Phase Microextraction: An Appealing Alternative for 
the Determination of Endogenous Substances - A Review. Anal. Chim. Acta. 2019, 1077, 67-86. DOI: 10.1016/j.aca.2019.05.054.

[11] Kataoka, H.; Saito, K. Recent Advances in SPME Techniques in Biomedical Analysis. J. Pharm. Biomed. Anal. 2011, 54, 926-950. DOI: 10.1016/j.jpba.2010.12.010.

[12] Pereira, J.; Silva, C. L.; Perestrelo, R.; Gonçalves, J.; Alves, V.; Câmara, J. S. Re-Exploring the High-Throughput Potential of Microextraction Techniques, SPME and MEPS, as Powerful Strategies for Medical Diagnostic Purposes. Innovative Approaches, Recent Applications and Future Trends. Anal. Bioanal. Chem. 2014, 406, 2101-2122. DOI: 10.1007/s00216013-7527-4.

[13] Hu, Y.; Pan, J.; Zhang, K.; Lian, H.; Li, G. Novel Applications of Molecularly-Imprinted Polymers in Sample Preparation. TrAC - Trends Anal. Chem. 2013, 43, 37-52. DOI: 10.1016/j. trac.2012.08.014.

[14] Behbahani, M.; Hassanlou, P. G.; Amini, M. M.; Moazami, H. R.; Abandansari, H. S.; Bagheri, A.; Zadeh, S. H. Selective Solid-Phase Extraction and Trace Monitoring of Lead Ions in Food and Water Samples Using New Lead-Imprinted Polymer Nanoparticles. Food Anal. Methods 2015, 8, 558-568. DOI: 10.1007/s12161-014-9924-5.

[15] Pardeshi, S.; Singh, S. K. Precipitation Polymerization: A Versatile Tool for Preparing Molecularly Imprinted Polymer Beads for Chromatography Applications. RSC Adv. 2016, 6, 23525-23536. DOI: 10.1039/C6RA02784A.

[16] Wackerlig, J.; Lieberzeit, P. A. Molecularly Imprinted Polymer Nanoparticles in Chemical Sensing - Synthesis, Characterisation and Application. Sensors Actuators B. Chem. 2015, 207, 144-157. DOI: 10.1016/j.snb.2014.09.094.

[17] Chen, L.; Wang, X.; Lu, W.; Wu, X.; Li, J. Molecular Imprinting: Perspectives and Applications. Chem. Soc. Rev. 2016, 45, 2137-2211. DOI: 10.1039/C6CS00061D.

[18] Fan, W.; He, M.; You, L.; Zhu, X.; Chen, B.; Hu, B. WaterCompatible Graphene Oxide/Molecularly Imprinted Polymer Coated Stir Bar Sorptive Extraction of Propranolol from Urine Samples Followed by High Performance Liquid Chromatography-Ultraviolet Detection. J. Chromatogr. A. 2016, 1443, 1-9. DOI: 10.1016/j.chroma.2016.03.017.

[19] Zhou, T.; Ding, L.; Che, G.; Jiang, W.; Sang, L. Recent Advances and Trends of Molecularly Imprinted Polymers for Specific Recognition in Aqueous Matrix: Preparation and Application in Sample Pretreatment. TrAC Trends Anal. Chem. 2019, 114, 11-28. DOI: 10.1016/j.trac.2019.02.028.

[20] Dabrowski, M.; Lach, P.; Cieplak, M.; Kutner, W. Nanostructured Molecularly Imprinted Polymers for Protein Chemosensing. Biosens. Bioelectron. 2018, 102, 17-26. DOI: 10.1016/j.bios.2017.10.045.

[21] Ansari, S.; Masoum, S. Molecularly Imprinted Polymers for Capturing and Sensing Proteins: Current Progress and Future Implications. TrAC - Trends Anal. Chem. 2019, 114, 29-47. DOI: $10.1016 /$ j.trac.2019.02.008.

[22] Xiao, D.; Jiang, Y.; Bi, Y. Molecularly Imprinted Polymers for the Detection of Illegal Drugs and Additives: A Review. Microchim. Acta 2018, 185, 247. DOI: 10.1007/s00604-0182735-4.

[23] Bossi, A. M.; Sharma, P. S.; Montana, L.; Zoccatelli, G.; Laub, O.; Levi, R. Fingerprint-Imprinted Polymer: Rational Selection of Peptide Epitope Templates for the Determination of Proteins by Molecularly Imprinted Polymers. Anal. Chem. 2012, 84, 4036-4041. DOI: 10.1021/ac203422r.

[24] Baydemir, G.; Denizli, A. Heparin Removal from Human Plasma Using Molecular Imprinted Cryogels. Artif. Cells. Nanomed. Biotechnol. 2015, 43, 403-412. DOI: 10.3109/ 21691401.2014.897631.

[25] Wang, J.; Wang, Q.-M.; Tian, L.-L.; Yang, C.; Yu, S.-H.; Yang, C. Research Progress of the Molecularly Imprinted Cryogel. Chinese J. Anal. Chem. 2015, 43, 1777-1784. DOI: 10.1016/ S1872-2040(15)60878-7.
[26] Topçu, A. A.; Bereli, N.; Albayrak, İ.; Denizli, A. Creatinine Imprinted Poly(Hydroxyethyl Methacrylate) Based Cryogel Cartridges. J. Macromol. Sci. Part A Pure Appl. Chem. 2017, 54, 495-501. DOI: 10.1080/10601325.2017.1320756.

[27] Dias, A. C. B.; Figueiredo, E. C.; Grassi, V.; Zagatto, E. A. G.; Arruda, M. A. Z. Molecularly Imprinted Polymer as a Solid Phase Extractor in Flow Analysis. Talanta 2008, 76, 988-996. DOI: 10.1016/j.talanta.2008.05.040.

[28] Iacob, B.-C.; Bodoki, A. E.; Oprean, L.; Bodoki, E. Metal-Ligand Interactions in Molecular Imprinting. In Ligand; InTech: London, UK, 2018. DOI: 10.5772/intechopen.73407.

[29] Tiwari, A.; Uzun, L.; Advanced Molecularly Imprinting Materials; Tiwari, A. Uzun, L., Eds.; John Wiley \& Sons, Inc.: Hoboken, NJ, 2016. DOI: 10.1002/9781119336181.

[30] Liu, Z.; He, H. Synthesis and Applications of Boronate Affinity Materials: From Class Selectivity to Biomimetic Specificity. Acc. Chem. Res. 2017, 50, 2185-2193. DOI: 10.1021/acs.accounts. $7 \mathrm{~b} 00179$.

[31] Bie, Z.; Chen, Y.; Ye, J.; Wang, S.; Liu, Z. Boronate-Affinity Glycan-Oriented Surface Imprinting: A New Strategy to Mimic Lectins for the Recognition of an Intact Glycoprotein and Its Characteristic Fragments. Angew. Chem. Int. Ed. Engl. 2015, 54, 10211-10215. DOI: 10.1002/anie.201503066.

[32] Wang, S.; Ye, J.; Bie, Z.; Liu, Z. Affinity-Tunable Specific Recognition of Glycoproteins via Boronate Affinity-Based Controllable Oriented Surface Imprinting. Chem. Sci. 2014, 5, 1135-1140. DOI: 10.1039/c3sc52986j.

[33] Xing, R.; Wang, S.; Bie, Z.; He, H.; Liu, Z. Preparation of Molecularly Imprinted Polymers Specific to Glycoproteins, Glycans and Monosaccharides via Boronate Affinity Controllable-Oriented Surface Imprinting. Nat. Protoc. 2017, 12, 964-987. DOI: 10.1038/nprot.2017.015.

[34] Huang, J.; Wu, Y.; Cong, J.; Luo, J.; Liu, X. Selective and Sensitive Glycoprotein Detection via a Biomimetic Electrochemical Sensor Based on Surface Molecular Imprinting and Boronate-Modified Reduced Graphene Oxide. Sensors Actuators B. Chem. 2018, 259, 1-9. DOI: 10.1016/j. snb.2017.12.049.

[35] de Faria, H. D.; Abrão, L. C.; de, C.; Santos, M. G.; Barbosa, A. F.; Figueiredo, E. C. New Advances in Restricted Access Materials for Sample Preparation: A Review. Anal. Chim. Acta. 2017, 959, 43-65. DOI: 10.1016/j.aca.2016.12.047.

[36] Moraes, G.; de, O. I.; Silva, L. M. R.; da, Santos-Neto, Á. J.; dos, Florenzano, F. H.; Figueiredo, E. C.; de. A New Restricted Access Molecularly Imprinted Polymer Capped with Albumin for Direct Extraction of Drugs from Biological Matrices: The Case of Chlorpromazine in Human Plasma. Anal. Bioanal. Chem. 2013, 405, 7687-7696. DOI: 10.1007/s00216-013-7275-5.

[37] Nazario, C. E. D.; Fumes, B. H.; da Silva, M. R.; Lanças, F. M. New Materials for Sample Preparation Techniques in Bioanalysis. J. Chromatogr. B. Analyt. Technol. Biomed. Life. Sci. 2017, 1043, 81-95. DOI: 10.1016/j.jchromb.2016.10.041.

[38] Urraca, J. L.; Aureliano, C. S. A.; Schillinger, E.; Esselmann, H.; Wiltfang, J.; Sellergren, B. Polymeric Complements to the Alzheimer's disease biomarker $\beta$-amyloid isoforms $\mathrm{A} \beta 1-40$ and $\mathrm{A} \beta 1-42$ for blood serum analysis under denaturing conditions. J. Am. Chem. Soc. 2011, 133, 9220-9223. DOI: 10.1021/ja202908z.

[39] Bousoumah, R.; Antignac, J. P.; Camel, V.; Grimaldi, M.; Balaguer, P.; Courant, F.; Bichon, E.; Morvan, M. L.; Le Bizec, B. Development of a Molecular Recognition Based Approach for Multi-Residue Extraction of Estrogenic Endocrine Disruptors from Biological Fluids Coupled to Liquid Chromatography-Tandem Mass Spectrometry Measurement. Anal. Bioanal. Chem. 2015, 407, 8713-8723. DOI: 10.1007/ s00216-015-9024-4.

[40] Li, D.; Yuan, Q.; Yang, W.; Yang, M.; Li, S.; Tu, T. Efficient Vitamin B12-Imprinted Boronate Affinity Magnetic Nanoparticles for the Specific Capture of Vitamin B12. Anal. Biochem. 2018, 561-562, 18-26. DOI: 10.1016/j.ab.2018.09.009. 
[41] Becerra, C. G.; Baez, F.; Lucangioli, S.; Flor, S.; Tripodi, V. Miniaturized Imprinted Solid Phase Extraction to the Selective Analysis of Coenzyme Q10 in Urine. J. Chromatogr. B. Analyt. Technol. Biomed. Life. Sci. 2019, 1116, 24-29. DOI: 10.1016/j. jchromb.2019.03.029.

[42] Lei, Y.; Xu, G.; Wei, F.; Yang, J.; Hu, Q. Preparation of a Stir Bar Coated with Molecularly Imprinted Polymer and Its Application in Analysis of Dopamine in Urine. J. Pharm. Biomed. Anal. 2014, 94, 118-124. DOI: 10.1016/j.jpba.2014.01. 041.

[43] de Oliveira, H. L.; Pires, B. C.; Teixeira, L. S.; Dinali, L. A. F.; Simões, N. S.; Borges, W.; de, S.; Borges, K. B. Novel Restricted Access Material Combined to Molecularly Imprinted Polymer for Selective Magnetic Solid-Phase Extraction of Estrogens from Human Urine. Microchem. J. 2019, 149, 104043. DOI: 10.1016/ j.microc.2019.104043.

[44] Bie, Z.; Xing, R.; He, X.; Ma, Y.; Chen, Y.; Liu, Z. Precision Imprinting of Glycopeptides for Facile Preparation of GlycanSpecific Artificial Antibodies. Anal. Chem. 2018, 90, 9845-9852. DOI: 10.1021/acs.analchem.8b01903.

[45] Bagán, H.; Zhou, T.; Eriksson, N. L.; Bülow, L.; Ye, L. and Characterization of Epitope-Imprinted Polymers for Purification of Human Hemoglobin. RSC Adv. 2017, 7, 41705-41712. DOI: 10.1039/C7RA07674F.

[46] Cenci, L.; Piotto, C.; Bettotti, P.; Bossi, A. M. Study on Molecularly Imprinted Nanoparticle Modified Microplates for Pseudo-ELISA Assays. Talanta 2018, 178, 772-779. DOI: 10.1016/j.talanta.2017.10.018.

[47] Li, S.; Yang, K.; Liu, J.; Jiang, B.; Zhang, L.; Zhang, Y. SurfaceImprinted Nanoparticles Prepared with a His-Tag-Anchored Epitope as the Template. Anal. Chem. 2015, 87, 4617-4620. DOI: $10.1021 /$ ac5047246.

[48] Madhumanchi, S.; Jadda, R.; Suedee, R. Efficient Adsorptive Extraction Materials by Surface Protein-Imprinted Polymer over Silica Gel for Selective Recognition/Separation of Human Serum Albumin from Urine. J. Appl. Polym. Sci. 2019, 136, 46894. DOI: 10.1002/app.46894.

[49] Liu, L.; Zhong, T.; Xu, Q.; Chen, Y. Efficient Molecular Imprinting Strategy for Quantitative Targeted Proteomics of Human Transferrin Receptor in Depleted Human Serum. Anal. Chem. 2015, 87, 10910-10919. DOI: 10.1021/acs.analchem. 5 b02633.

[50] Bertolla, M.; Cenci, L.; Anesi, A.; Ambrosi, E.; Tagliaro, F.; Vanzetti, L.; Guella, G.; Bossi, A. M. Solvent-Responsive Molecularly Imprinted Nanogels for Targeted Protein Analysis in MALDI-TOF Mass Spectrometry. ACS Appl. Mater. Interfaces 2017, 9, 6908-6915. DOI: 10.1021/acsami.6b16291.

[51] Xu, J.; Ambrosini, S.; Tamahkar, E.; Rossi, C.; Haupt, K.; Tse Sum Bui, B. Toward a Universal Method for Preparing Molecularly Imprinted Polymer Nanoparticles with Antibodylike Affinity for Proteins. Biomacromolecules 2016, 17, 345-353. DOI: $10.1021 /$ acs.biomac.5b01454.

[52] Mergola, L.; Orabona, C.; Albini, E.; Vasapollo, G.; Scorrano, S.; Del Sole, R. Urinary L-Kynurenine Quantification and Selective Extraction through a Molecularly Imprinted Solid-Phase Extraction Device. J. Sep. Sci. 2018, 41, 3204-3212. DOI: 10.1002/jssc.201800458.

[53] Ertürk, G.; Bereli, N.; Ramteke, P. W.; Denizli, A. Molecularly Imprinted Supermacroporous Cryogels for Myoglobin Recognition. Appl. Biochem. Biotechnol. 2014, 173, 1250-1262. DOI: 10.1007/s12010-014-0844-z.

[54] Dolak, İ.; Keçili, R.; Onat, R.; Ziyadanoğulları, B.; Ersöz, A.; Say, R. Molecularly Imprinted Affinity Cryogels for the Selective Recognition of Myoglobin in Blood Serum. J. Mol. Struct. 2018, 1174, 171-176. DOI: 10.1016/j.molstruc.2018. 03.126.

[55] Wen, L.; Tan, X.; Sun, Q.; Svec, F.; Lv, Y. "Smart" molecularly imprinted monoliths for the selective capture and easy release of proteins. J. Sep. Sci. 2016, 39, 3267-3273. DOI: 10.1002/jssc. 201600576.
[56] Qader, A. A.; Urraca, J.; Torsetnes, S. B.; Tønnesen, F.; Reubsaet, L.; Sellergren, B. Peptide Imprinted Receptors for the Determination of the Small Cell Lung Cancer Associated Biomarker Progastrin Releasing Peptide. J. Chromatogr. A. 2014, 1370, 56-62. DOI: 10.1016/j.chroma.2014.10.023.

[57] Demirci, B.; Bereli, N.; Aslıüce, S.; Baydemir, G.; Denizli, A. Protein C Recognition by Ion-Coordinated Imprinted Monolithic Cryogels. J. Sep. Sci. 2017, 40, 1610-1620. DOI: $10.1002 /$ jssc. 201600992.

[58] Xu, L.; Hu, Y.; Shen, F.; Li, Q.; Ren, X. Specific Recognition of Tyrosine-Phosphorylated Peptides by Epitope Imprinting of Phenylphosphonic Acid. J. Chromatogr. A. 2013, 1293, 85-91. DOI: 10.1016/j.chroma.2013.04.013.

[59] Li, D.; Bie, Z.; Wang, F.; Guo, E. Efficient Synthesis of Riboflavin-Imprinted Magnetic Nanoparticles by Boronate Affinity-Based Surface Imprinting for the Selective Recognition of Riboflavin. Analyst 2018, 143, 4936-4943. DOI: 10.1039/ c8an01044g.

[60] Xu, J.; Haupt, K.; Tse Sum Bui, B. Core-Shell Molecularly Imprinted Polymer Nanoparticles as Synthetic Antibodies in a Sandwich Fluoroimmunoassay for Trypsin Determination in Human Serum. ACS Appl. Mater. Interfaces 2017, 9, 24476-24483. DOI: 10.1021/acsami.7b05844.

[61] Turan, E. His-Tag-Epitope Imprinted Thermoresponsive Magnetic Nanoparticles for Recognition and Separation Thyroid Peroxidase Antigens from Whole Blood Samples. ChemSelect 2018, 3, 11963-11969. DOI: 10.1002/slct.201801557.

[62] Chianella, I.; Guerreiro, A.; Moczko, E.; Caygill, J. S.; Piletska, E. V.; De Vargas Sansalvador, I. M. P.; Whitcombe, M. J.; Piletsky, S. A. Direct Replacement of Antibodies with Molecularly Imprinted Polymer Nanoparticles in ELISAdevelopment of a novel assay for vancomycin. Anal. Chem. 2013, 85, 8462-8468. DOI: 10.1021/ac402102j.

[63] Tang, J.; Wang, J.; Shi, S.; Hu, S.; Yuan, L. Determination of $\beta$-Agonist Residues in Animal-Derived Food by a Liquid Chromatography-Tandem Mass Spectrometric Method Combined with Molecularly Imprinted Stir Bar Sorptive Extraction. J. Anal. Methods Chem. 2018, 2018, 9053561-9053510. DOI: $10.1155 / 2018 / 9053561$.

[64] Luo, X.; Li, G.; Hu, Y. In-Tube Solid-Phase Microextraction Based on NH2-MIL-53(Al)-polymer monolithic column for online coupling with high-performance liquid chromatography for directly sensitive analysis of estrogens in human urine. Talanta 2017, 165, 377-383. DOI: 10.1016/j.talanta.2016.12.050.

[65] Costa Queiroz, M. E.; Donizeti de Souza, I.; Marchioni, C. Current Advances and Applications of in-Tube Solid-Phase Microextraction. TrAC - Trends Anal. Chem. 2019, 111, 261-278. DOI: 10.1016/j.trac.2018.12.018.

[66] Lequin, R. M. Enzyme Immunoassay (EIA)/Enzyme-Linked Immunosorbent Assay (ELISA). Clin. Chem. 2005, 51, 2415-2418. DOI: 10.1373/clinchem.2005.051532.

[67] Moczko, E.; Richard, D.; Bernabé, R.; Camilo, G.; Eduardo, P.; Sergey, P.; César, C. Molecularly Imprinted Nanoparticles Assay (MINA) in Pseudo ELISA: An Alternative to Detect and Quantify Octopamine InWater and Human Urine Samples. Polymers (Basel) 2019, 11, 1497-1413. DOI: 10.3390/ polym11091497.

[68] Bi, X.; Liu, Z. Facile Preparation of Glycoprotein-Imprinted 96Well Microplates for Enzyme-Linked Immunosorbent Assay by Boronate Affinity-Based Oriented Surface Imprinting. Anal. Chem. 2014, 86, 959-966. DOI: 10.1021/ac403736y.

[69] Cáceres, C.; Canfarotta, F.; Chianella, I.; Pereira, E.; Moczko, E.; Esen, C.; Guerreiro, A.; Piletska, E.; Whitcombe, M. J.; Piletsky, S. A. Does Size Matter? Study of Performance of PseudoELISAs Based on Molecularly Imprinted Polymer Nanoparticles Prepared for Analytes of Different Sizes. Analyst 2016, 141, 1405-1412. DOI: 10.1039/c5an02018b.

[70] Shi, W.; Zhang, S. Q.; Li, K.; Bin; Jia, W. P.; Han, D. M. Integration of Mixed-Mode Chromatography and Molecular Imprinting Technology for Double Recognition and Selective 
Separation of Proteins. Sep. Purif. Technol. 2018, 202, 165-173. DOI: 10.1016/j.seppur.2018.03.057.

[71] Yang, C.; Liu, Y.-R.; Zhang, Y.; Wang, J.; Tian, L.-L.; Yan, Y.N.; Cao, W.-Q.; Wang, Y.-Y. Depletion of Abundant Human Serum Proteins by per Se Imprinted Cryogels Based on Sample Heterogeneity. Proteomics 2017, 17, 1600284. DOI: 10.1002/ pmic.201600284.

[72] Santos, M. G.; Tavares, I. M. C.; Barbosa, A. F.; Bettini, J.; Figueiredo, E. C. Analysis of Tricyclic Antidepressants in Human Plasma Using Online-Restricted Access Molecularly Imprinted Solid Phase Extraction Followed by Direct Mass Spectrometry Identification/Quantification. Talanta 2017, 163, 8-16. DOI: 10.1016/j.talanta.2016.10.047.

[73] Jagadeesan, K. K.; Rossetti, C.; Abdel Qader, A.; Reubsaet, L.; Sellergren, B.; Laurell, T.; Ekström, S. Filter Plate-Based Screening of MIP SPE Materials for Capture of the Biomarker
Pro-Gastrin-Releasing Peptide. SLAS Discov. 2017, 22, 1253-1261. DOI: 10.1177/2472555216689494.

[74] Aebersold, R.; Mann, M. Mass-Spectrometric Exploration of Proteome Structure and Function. Nature 2016, 537, 347-355. DOI: $10.1038 /$ nature 19949 .

[75] Zhao, Y. Sequence-Selective Recognition of Peptides in Aqueous Solution: A Supramolecular Approach through Micellar Imprinting. Chemistry 2018, 24, 14001-14009. DOI: 10.1002/chem.201801401.

[76] Awino, J. K.; Zhao, Y. Imprinted Micelles for Chiral Recognition in Water: Shape, Depth, and Number of Recognition Sites. Org. Biomol. Chem. 2017, 15, 4851-4858. DOI: $10.1039 / \mathrm{c} 7 \mathrm{ob} 00764 \mathrm{~g}$.

[77] Abrão, L. C. C.; Figueiredo, E. C. A New Restricted Access Molecularly Imprinted Fiber for Direct Solid Phase Microextraction of Benzodiazepines from Plasma Samples. Analyst 2019, 144, 4320-4330. DOI: 10.1039/C9AN00444K. 